\title{
Study on the Effect of Distraction for Pedestrian Crossing
}

\author{
Han Li ${ }^{1, a}$, Xiaosong Ming ${ }^{1, b}$ \\ ${ }^{1}$ 2\#, Sipailou, Nanjing, Jiangsu, China \\ alhfrostring@126.com, b220142608@seu.edu.cn
}

\begin{abstract}
Keywords: Pedestrian Safety; Distraction; Pedestrian; Statistical Analysis.
\end{abstract}
Abstract. As for the traffic hidden danger generated by pedestrians when they are distracted while crossing the street, this study selects the typical area of the signalized and unsignalized crosswalk collects the data of them by artificial investigation and draws the conclusions. It is concluded that on the two types of crosswalks, people on the signalized crosswalks are more easily distracted. For different age groups of pedestrians, young people are more likely to have distraction behavior than middle aged people and old people. For different behaviors of distraction, the risk of traffic accidents from high to low are generated by using mobile phones to surf the Internet, making phone calls, listening to music, and concentrating on crossing the street. The study provides data support and basis for traffic management department to improve pedestrian safety.

\section{Introduction}

Road traffic system is composed of five elements, including people, cars, roads, environment and management, among which pedestrian is the largest number of traffic systems and the most irregular component, and compared with motor vehicles and non-motor vehicles, pedestrians are the weak in traffic activities. When pedestrians and vehicles have an accident, the injured is often pedestrians; the main cause of the traffic accident is that pedestrians do not observe the traffic rules, or they play mobile phones, make phone calls, send text messages, or listen to music when they walk or walk through the streets, which in the end leads to traffic accidents because of their distraction. With the development of the city, traffic development places more emphasis on "people-oriented", in transportation planning, design and management, people need to have more consideration of vulnerable groups. Therefore, it is necessary to find out the relationship between pedestrian safety and distraction, thus reducing the pedestrian traffic accidents.

Foreign experts have made in-depth studies on pedestrian distraction [1-6], at the same time, they have made studies on different groups of people. The main research idea is to select the research object in the way of random sampling, then to further understand the walking habits, gender, age, race and so on of the research object, and carry out training on experiment courses. And then the research subjects are divided into different groups, and get the evaluation index of the safe crossing through the simulation of crossing behavior in virtual pedestrian environment. At last, the data is analyzed by the method of logistic regression and two element regression and other methods, thus finding out what kind of behavior influence greatly on pedestrian crossing safety. The operation of this research method is convenient but it has much occasional climatic factors. The studies of domestic scholars do not involve the influence of distraction on pedestrian crossing safety, and their studies on pedestrian crossing time, speed and psychological cognitive process are all made by watching pedestrians cross the street in their normal way, and the analysis of the impact of pedestrian safety reasons is from macro perspective [7-11], which does not relate to the specific impact of distraction on pedestrian safety.

Therefore this study can learn from foreign research methods in this area, combine with the existing research results in China, and drill down further to determine exactly the influence degree of various factors affecting pedestrian concentration in different street environment. 


\section{Survey Data}

Survey Location Selection. According to the distribution characteristics of China pedestrian crossing place, usually, the intersections with large amount of pedestrians are as follows:1) intersection near the station pier; 2) the intersection of a theater, a cinema, a park and a playground;3) the intersection near school and residential areas;4) the intersection near a busy commercial area. This paper is to investigate the effects of dispersion and crossing for pedestrians attention, and generally in the intersection near the pedestrian street and the school, pedestrians' scattered attention behavior occurs more often. Therefore, in this study, signalized crosswalk is set in front of Hubei Provincial Traditional Chinese Medical Hospital, which is at Luo Yu Road of Wuhan City, while unsignalized crosswalk is set in Optics Valley Wuhan Square Ring Intersection.

Data Collection. The data acquisition is based on manual counting method; data acquisition time is from 14:00 to 15:00 at every working day. Signal lamp configuration for the four phase, the signal period is $106 \mathrm{~s}$, the signal period for turning left to the hospital of traditional Chinese medicine is $13 \mathrm{~s}$, and people can go straight in the westbound direction, and the signal period for going straight to the East is $63 \mathrm{~s}$, and that for pedestrians to pass the street is $24 \mathrm{~s}$, yellow light time at per phase is $3 \mathrm{~s}$.

\section{Analysis of the Characteristics of Attention Distraction Behavior}

We get the number of pedestrian crossing behavior and related data through field survey, the data is analyzed further with the method of mathematical statistics, and thus we can better understand the relationship of pedestrian crossing distraction behavior with different types of pedestrian crosswalk, gender and age groups.

Analysis of distraction behavior and control method of pedestrian crossing control mode. Through Fig 1, we can see that there are three kinds of behavior of distraction, they refer to make a phone call, mobile Internet and listening to music. At the same time, the number of pedestrian distraction in signalized crosswalk accounted for $22.9 \%$ of the total number, accounted for $12.5 \%$ at the unsignalized crosswalk, all accounted for a large proportion. The proportion of pedestrian distraction at signalized crosswalk is higher than that at the non signalized crosswalk, the reason is that pedestrians believe that the vehicles and pedestrians are separated in time in the case of signal control, so the street is relatively safe; while at the unsignalized crosswalk, pedestrians should always watch out for vehicles, so their attention will be more concentrated.

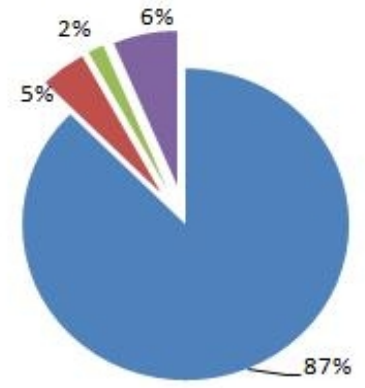

None
Surfing the Internet
Listening to music a) Signalized crosswalk
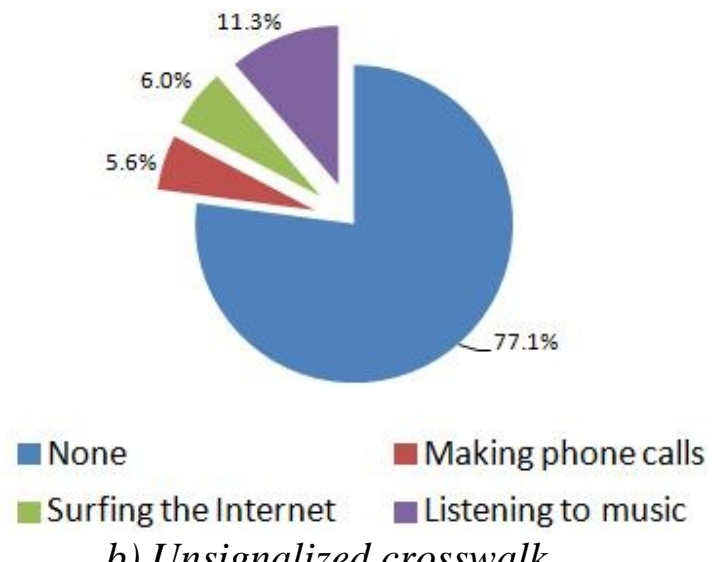

b) Unsignalized crosswalk

Fig.1 Proportion of pedestrian crosswalk distracted behavior

Analysis of pedestrian distraction behavior and gender. The survey has found that pedestrians of different gender have different behavior on the street, distraction behavior of pedestrians with different genders can be obtained through statistics. It is indicated that the number of male distraction behaviors was slightly higher than that of female at signalized crosswalk, because men are more flexible than women, and their reactions are more agile, thus they are casual when they are waiting to cross the street; while the number of male distraction behaviors was slightly lower than that of female 
at non signalized crosswalk, this is because that women are more cautious, and they would rather wait than rush to cross the street if they believe that the crossing is not safe.

Analysis of pedestrian distraction behavior and age. We can find from the survey (Fig 2), young people's attention distraction behavior both at signalized crosswalk and at non signalized crosswalk is very prominent, accounting for the vast majority, the main reason is that young people's preferences for electronic products are much higher than those of other age groups. While middle aged people are more mature than young people, and their degree of dependence on electronic products is not high, therefore, the number of people who have distraction behavior is relatively small, and their distraction behavior is mainly to make phone calls; there is almost no telephone, listening to music and other distracting behavior for the aged while they cross the street.

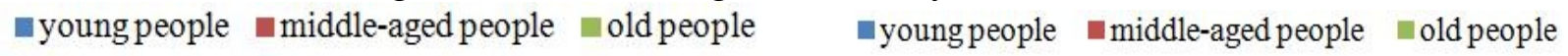

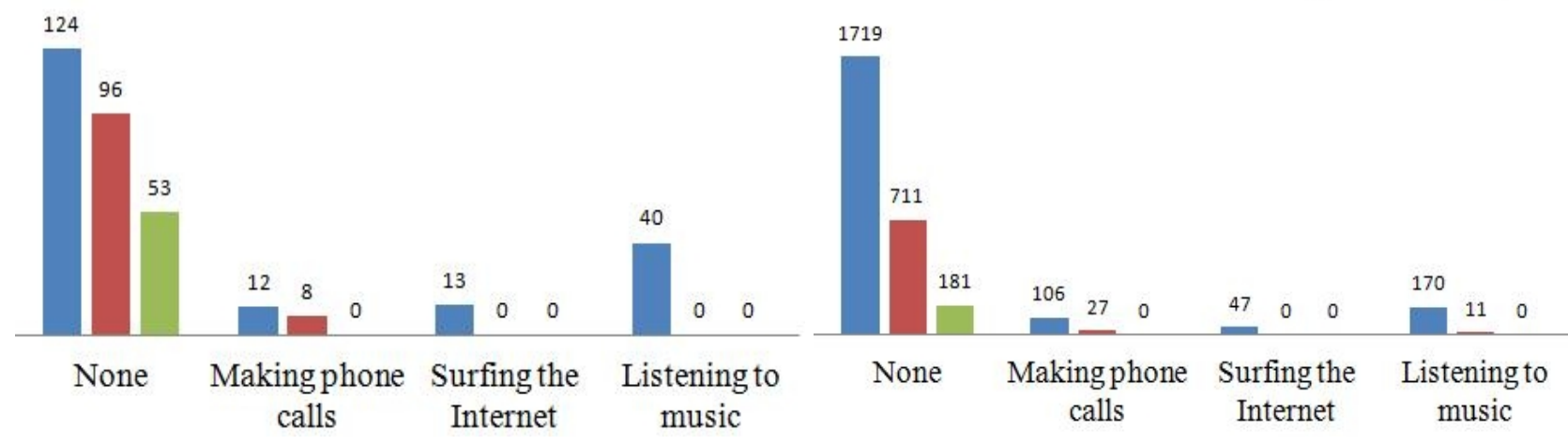

a) Signalized crosswalk b) Unsignalized crosswalk

Fig.2 Crosswalk pedestrian distraction feature of different ages

Analysis of pedestrian distraction behavior and crossing speed. By recording the different types of pedestrian crossing time, then take the average value, we can obtain the average crossing time under different behavior, and according to the average crossing time, we can reach the average speed. As shown in Fig 3, pedestrian's crossing speed is higher at signalized crosswalk, the reason is that pedestrians have to avoid the vehicles at non signalized crosswalk, thus causing pedestrian crossing delay. Pedestrian distraction behavior from high to low are generated by using mobile phones to surf the Internet, making phone calls, listening to music, and concentrating on crossing the street, because pedestrians focus on using mobile phones while ignoring the surrounding environment changes, in other behaviors, pedestrians can walk in a relatively normal speed while making phone calls or listening to music.

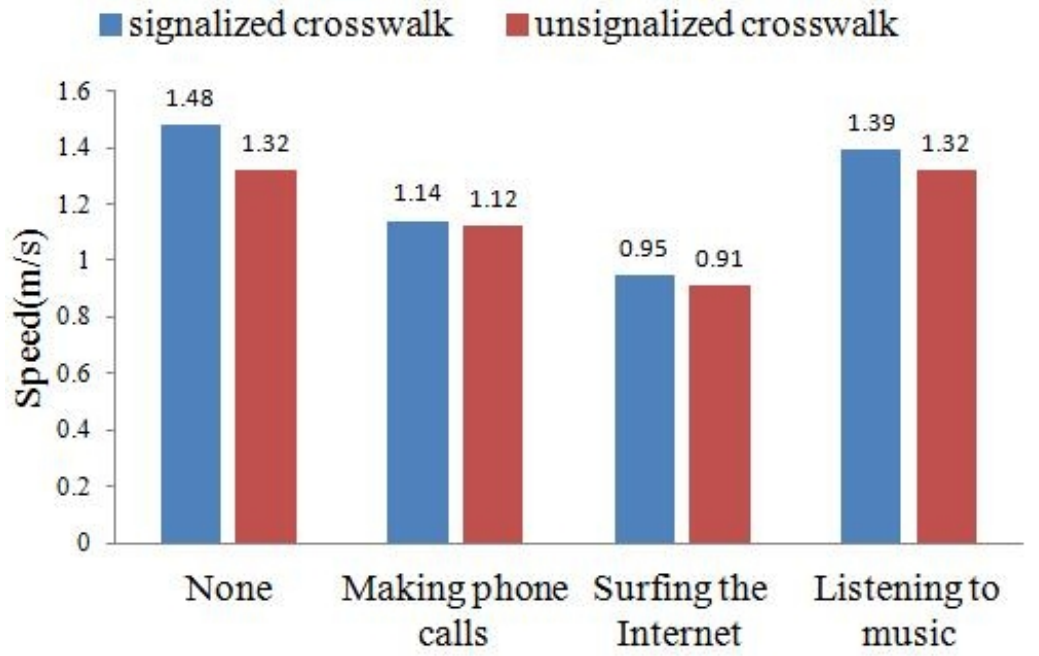

Fig.3 Diagram of pedestrian crossing speed 


\section{Conclusions}

This study obtains pedestrian behavior characteristics and related parameters through field investigation, and it finds out that people on the signalized pedestrian crossing are more easily distracted, among which mobile Internet is more likely to lead to traffic accidents, especially young people are more likely to be distracted, and this not only affects the efficiency of pedestrian crossing, but also produces a traffic safety hazard. therefore, the safety situation while pedestrian crossing the street is eager to be improved, research results provide data support and basis for traffic management department to improve pedestrian safety. On the one hand, the traffic management department should strengthen the propaganda and education of traffic safety, especially the safety education for the majority of teenagers, and let them to know that the phone call and mobile Internet when they walk across the street will cause great interference to traffic, at the same time, these actions will threaten their lives; on the other hand, warning signs can also be set up at crosswalks to remind pedestrians the danger of mobile Internet when they walk across the street.

\section{References}

[1] K.W. Byington, D.C. Schwebel: Effects of mobile Internet use on college student pedestrian injury risk. Accident Analysis \& Prevention Vol.51 (2012), p. 78-83.

[2] L.L. Thompson, F.P. Rivara, R.C. Ayyagari and B.E. Ebel: Impact of social and technological distraction on pedestrian crossing behaviour: an observational study. Injury prevention Vol.19(2013), p. 232-237.

[3] D.C. Schwebel, D. Stavrinos, K.W. Byington, T. Davis, E.E. O’Neal, D. Jong: Distraction and pedestrian safety: How talking on the phone, texting, and listening to music impact crossing the street. Accident Analysis \& Prevention Vol.45 (2012), p.266-271.

[4] E.M. Lamberg, L.M. Muratori: Cell phones change the way we walk. Gait \& Posture Vol.35 (2012), p. 688-690.

[5] J.L. Nasa, D. Troyer: Pedestrian injuries due to mobile phone use in public places. Accident Analysis \& Prevention Vol.57 (2013), p.91-95.

[6] M.B. Neider, J.S. McCarley, J.A. Crowell, H. Kaczmarski, A.F. Kramer: Pedestrians, vehicles, and cell phones. Accident Analysis \& Prevention Vol.42 (2010), p.589-594.

[7] C. Jing: Study on Pedestrian Crossing Characteristics. Changchun: Jilin University, 2007.

[8] L. Zhao: Study on Pedestrian Crossing Characteristics at Signalized Intersection. Beijing: Beijing University of Technology, 2007.

[9] X. Yang, Y. Lao, M. Yun: Application of Different Pedestrian Cross Pattern to No-Signal Controlled Segment. Journal of Tongji University Vol.35 (2007), p.1466-1470.

[10] Y. Pei, S. Feng: Research on Design Speed of Urban Pedestrian Crossing. Journal of Highway and Transportation Research and Development Vol.23 (2006), p. 104-107.

[11] S. Feng, Y. Wu: Crossing speed analysis for pedestrian at signalized intersection. Journal of Harbin Institute of Technology Vol.36 (2004), p. 76-78. 